\title{
La Arquitectura como disciplina clave para el estudio del Hábitat Rural en climas áridos y (semi) desérticos ${ }^{1}$. El caso de Mendoza, Argentina.
}

\author{
Architecture as a key discipline for the study of Rural Habitat in arid and (semi) desert \\ climates. The case of Mendoza, Argentina.
}

Recibido:11/12/2017

Virginia Miranda Gassull ${ }^{2}$

Aprobado: 11/2/2018

Matías Esteves ${ }^{3}$

\section{Resumen:}

Los cambios del espacio rural especialmente zonas de clima (semi) desértico, están insertas en procesos de transformación y dominación de la naturaleza que repercuten en la configuración del hábitat. Desde esta perspectiva se establece la relación estrecha que existe con el campo de la arquitectura para pensar diseños metodológicos afines para su estudio. Por lo tanto, el objetivo es procurar una base de procedimientos metodológicos de abordaje del hábitat rural de zonas no irrigadas, basadas en la inclusión de diversos componentes que inciden en la disposición y análisis arquitectónico. La metodología empleada es la comparación de dos trabajos doctorales realizados en la misma área (provincia de Mendoza-Argentina). El resultado señala un diseño metodológico basado en la relación naturaleza-cultura y una segunda propuesta deriva de la relación entre el hábitat-territorio según la organización social de base. Se puede destacar que el uso de metodologías cualitativas desde la participación de la población local como actores claves en la construcción del hábitat y el territorio aporta a la comprensión del hábitat y la vivienda como un fenómeno complejo y no solo como objetos materiales. En ambos procedimientos el relevamiento arquitectónico fue clave en la construcción de datos sobre las viviendas y los asentamientos.

\section{Palabras Clave:}

Metodología; hábitat rural; arquitectura; tierras secas.

\begin{abstract}
:
The changes in the rural space, especially areas of (semi) desert climate, are inserted in processes of transformation and domination of nature that have an impact on the habitat configuration. From this perspective, the close relationship that exists with the field of architecture is established to think about related
\end{abstract}

\footnotetext{
${ }^{1}$ Éste trabajo se ha realizado con diversos datos de las tesis doctorales de los autores en Ordenamiento Territorial y en Arquitectura, financiados por el Consejo Macional de Investigaciones Científicas y Técnicas (CONICET).

${ }^{2}$ Nacionalidad: argentina. Doctora en Ordenamiento Territorial y Desarrollo Sostenible. Adscripción: Docente de la Facultad de Ingeniería en la Carrera de Arquitectura-Uncuyo(Catedra de Vivienda Social) Mendoza, Argentina. arq.vmiranda@gmail.com

${ }^{3}$ Nacionalidad: argentino. Doctor en Arquitectura-San Juan Mendoza. Adscripción: CONICET, Argentina. matiasesteves@hotmail.com
} 
methodological designs for their study. Therefore, the objective is to provide a base of methodological procedures to address the rural habitat of non-irrigated areas, based on the inclusion of various components that affect the layout and architectural analysis. The methodology used is the comparison of two doctoral works carried out in the same area (province of MendozaArgentina). The result indicates a methodological design based on the nature-culture relationship and a second proposal derives from the relationship between the habitat-territory according to the social organization of the base. It can be highlighted that the use of qualitative methodologies from the participation of the local population as key actors in the construction of habitat and territory contributes to the understanding of habitat and housing as a complex phenomenon and not only as material objects. In both procedures the architectural survey was key in the construction of data on housing and settlements.

\section{Key words:}

methodology; rural habitat; architecture; drylands. 


\section{Introducción}

Las transformaciones del mundo agrario rural son una realidad a la orden del día en Latinoamérica, que desde hace tres décadas se vienen consolidando diversos cambios apoyados en un modelo productivo de total dominación de la naturaleza (principalmente el agua y la tierra), donde inciden la globalización de los sistemas agroalimentarios, el uso de nuevas tecnologías y los cambios socioproductivos, entre otros (Teubal, 2001; Rodríguez y Meneses, 2011). En este sentido, la "ruralidad" de la provincia de Mendoza resulta fragmentada por el acceso y goce del derecho al agua asociado a la propiedad de la tierra, dispuesto por la Ley de Aguas que data de 1884 (Montaña, 2008). El derecho al agua sobre la tierra marca una gradual diferencia de valor mercantil productivo, social e histórico que ha incidido en la fragmentación del territorio, donde se encuentran sectores del territorio con derecho al agua oasis- $\mathrm{y}$ otros que no tienen acceso a agua y son denominados áreas rurales no irrigadas. El primero conforma el $3 \%$ de la superficie provincial mientras que el segundo representa el $97 \%$ de la superficie total (Gudiño, 2017).

En la provincia de Mendoza (Argentina) los procesos de transformación de los territorios rurales han estado asociados a la vitivinicultura en las zonas irrigadas, que desde la década de los 90 "han mutado de un patrón tradicional minifundista con derecho de riego a una vitivinicultura de exportación de vinos de alta calidad por capitales internacionales" (Martin, F. 2009:120). Este nuevo modelo, al igual que el anterior, deja por fuera a otros territorios rurales no aptos para la producción agrícola vitivinícola.
La exclusión de los sectores no irrigados implica como afirman Ivars J. y Larsimont,R. (2015) “una violencia epistémica explícitas e implícitas en las relaciones sociales entre productores desde la cuestión del acceso y uso del agua...() De este modo, la violencia epistémica aparece como una forma de invisibilizar al otro, expropiando su posibilidad de representación" (2015:8). A lo que se suma que la concentración de bienes naturales, población y poder en una acotada superficie del territorio provincial resulta en detrimento del agotamiento y despojo de los bienes naturales a grupos sociales minoritarios ubicados en los sectores no irrigados, aportando a su marginalidad y exclusión (Montaña et al, 2003).

Hay que destacar que según el criterio demográfico utilizado por el Instituto Nacional de Estadísticas y Censos -INDEC- de la Argentina, establece a lo rural a todas las localidades de menos de 2000 habitantes, sin embargo este criterio no diferencia la diversidad que hay en la "ruralidad". Resulta de interés destacar lo "rural no irrigado" como una categoría de trabajo específica que se diferencia ampliamente de los sectores agrícolas productivos que se encuentran en zonas irrigadas -oasisde la provincia de Mendoza. A lo que se suma que las tierras secas no irrigadas son mal llamadas "desierto", lo que profundiza su exclusión epistemológica dado que en los sectores no irrigados hay vida, hay una población criancera y en el caso de Mendoza ha sido y es en la actualidad también el hábitat de la población originaria. Por ello, se pretende indagar en las heterogeneidades que reviste el hábitat de las tierras secas no irrigadas, es decir no sólo en la mirada puesta en lo productivo como determinante de lo rural, sino también en las formas de la vida cotidiana poniendo en relieve a la arquitectura como disciplina de base para la construcción de datos. 
El cuestionamiento central del trabajo es: ¿Cómo podría abordarse el hábitat rural de tierras secas no irrigadas para dar cuenta de la riqueza de estos territorios? Se busca definir cuáles son los posibles caminos metodológicos para la obtención y análisis de datos. Cabe destacar que este trabajo lejos de indagar en una novedosa propuesta metodológica, se presenta como fruto de una serie de reflexiones efectuadas con motivo de los trabajos doctorales, relevamiento y catalogación llevados a cabo en la zona rural no irrigada del noreste de la provincia de Mendoza (Departamento de Lavalle), Argentina. Por ello, el objetivo del trabajo es procurar una base de procedimientos metodológicos de abordaje sobre el hábitat rural de zonas no irrigadas, basadas en la inclusión de los diversos componentes que inciden en su configuración y análisis arquitectónico con vistas a enriquecer el abordaje del hábitat en estos territorios.

Como resultado, este trabajo propone dos caminos válidos para abordar la problemática habitacional de los sectores rurales no irrigados. Las propuestas son alternativas metodológicas que lejos de ser caminos paralelos se pueden integrar, complementarse y conjugarse. La propuesta resulta afín de facilitar la obtención de datos para la comprensión del hábitat rural de tierras secas no irrigadas factibles de ser aplicadas tanto en el diseño de políticas públicas de índole habitacional o en el campo de los avances en investigación y en la academia.

\section{El territorio no irrigado de Mendoza}

La provincia de Mendoza se localiza en la denominada “diagonal árida sudamericana”, donde por la aridez de su clima sumado a las escasas precipitaciones que se registran-promedio de $250 \mathrm{~mm}$ anuales- las actividades agrícolas son dependientes del agua de los ríos. En torno de centros urbanos y campos para la explotación agrícola. Por fuera de los oasis se encuentran las tierras secas sin posibilidad de riego y con actividades productivas de subsistencia. Ver Figura 1.

FIGURA 1: MAPA DE LA PROVINCIA DE MENDOZA (DONDE SE OBSERVAN LOS OASIS

ARTIFICIALES DE RIEGO. SE SEÑALA LA UBICACIÓN DEL DEPARTAMENTO DE LAVALLE

EN EL NORESTE PROVINCIAL DONDE SE OBSERVA SU LOCALIZACIÓN PERIFÉRICA RESPECTO AL OASIS NORTE).

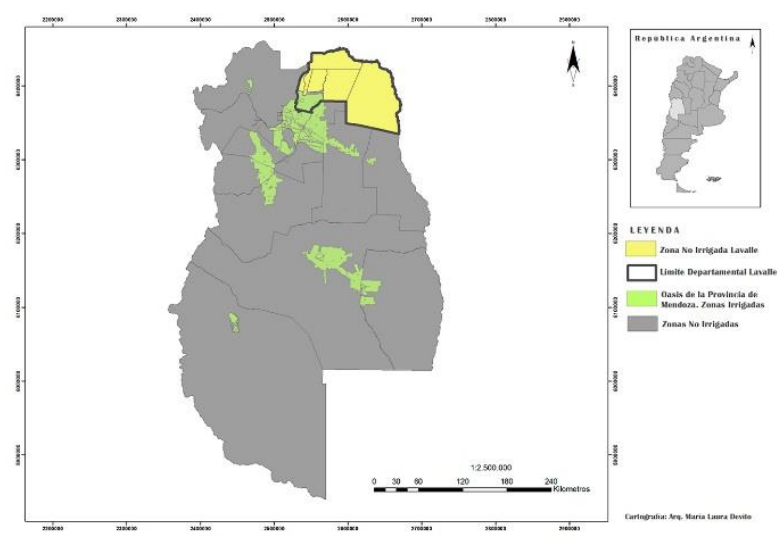

Fuente: cartografía realizada por María Laura Devito en base a información de elaboración propia, 2017

Conforme el crecimiento sostenido del oasis durante el siglo XIX y XX y el éxito de las actividades agrícolas centradas en la vitivinicultura, el aprovechamiento del caudal del rio en la parte media de la cuenca se intensificó, lo que implicó menor caudal de agua hacia la parte baja del rio, desproveyendo de agua superficial a esa zona. La falta de agua desencadenó procesos de desertificación que afectaron al ecosistema natural y a las comunidades Huarpes que habitan en esos territorios. La construcción del patrón territorial fragmentado irrigadono irrigado queda de relieve en el Plan Provincial de Ordenamiento Territorial (PPOT), donde se expresa que: 
"Las unidades territoriales descriptas demuestran que el factor clave ha sido y es la existencia de agua, la que explica el modelo de desarrollo que surge a fines del siglo XIX, en un territorio muy frágil por su clima y amenazas naturales, pero con muchas potencialidades en cuanto a la riqueza de recursos naturales y valores sociales y culturales que permitieron superar las adversidades que imponía el medio con trabajo y tesón. Se privilegia la vida en oasis de riego en donde se planifican ciudades y se desarrolla una agricultura intensiva. A más de un siglo de su existencia, el modelo no logra integrar las tierras no irrigadas y los procesos de concentración acentúan los desequilibrios territoriales, las iniquidades y el deterioro ambiental" (PPOT, 2016:22).

Actualmente, la provincia está inserta en un proceso de (re) ordenamiento territorial desde la sanción de la Ley $\mathrm{N}^{\circ} 8051$ en el 2009 de ordenamiento territorial de Mendoza y la elaboración del Plan Provincial de Ordenamiento Territorial en 2017. En este plan se define a las áreas rurales no irrigadas como:

"aquellas partes del territorio que se encuentran escasamente modificadas por la acción del hombre, es decir, no han sido objeto de mayores intervenciones humanas, se caracterizan por una lenta transformación del uso del suelo, poseen una alta demanda de recursos naturales escasos (agua, suelo, vegetación y otros). Su evolución ha sido espontánea y dependiendo del ambiente natural originario (...) Estas áreas representan uno de los mayores desafíos de la administración para corregir los desequilibrios territoriales..."
(Secretaría de Ambiente y Ordenamiento Territorial, 2017:71).

$\mathrm{Si}$ bien en esta definición se mencionan algunas características de las zonas no irrigadas, se omite que la mayor transformación de estos territorios se desencadenó por la falta de agua superficial, que fue aprovechada en la zona del oasis provocando los actuales procesos de desertificación que afectan a los pobladores locales. Históricamente, en la parte baja de la cuenca se encontraban lagunas de considerable tamaño reconocidas por diversos autores (Chiavazza, 2008; Roig et al, 1999) y relatos de viajeros (Rosales, 1877; Vicuña, 1856). En estos textos queda de manifiesto la biodiversidad que existía en el sector y la importancia de las lagunas para el desarrollo de la vida diaria de la población local, donde se registraban como actividades productivas principales la agricultura y la pesca. La falta de agua cada vez más evidente desde principios del siglo XX provocó la sequía de las lagunas y se propició actividades de subsistencia centrados en la cría de ganado caprino. La agricultura y la pesca ya no eran posibles por la falta de agua superficial.

La metodología empleada en este trabajo resulta de la discusión y comparación de dos trabajos doctorales que se realizaron en un mismo territorio $y$ fueron desarrolladas con diferentes procedimientos metodológicos tanto para el trabajo de campo como para la sistematización de datos, y se obtuvieron resultados similares. A partir de la exposición del análisis y construcción de indicadores y variables de cada propuesta, se exponen los puntos comunes, y se destaca el papel de la arquitectura como una disciplina clave para pensar diseños metodológicos en zonas rurales. 
Dentro del noreste provincial se trabajó en el departamento de Lavalle, puntualmente con los distritos de Lagunas del Rosario y La Asunción. Ambos distritos son atravesados por el Rio Mendoza, que desde el 2008 no tienen cotas de agua que rieguen el territorio. La población que habita este sector es predominantemente criancera caprina, la mayoría se autoreconoce como descendiente originario Huarpe y están organizados en 13 comunidades reconocidas por el Instituto Nacional Indígena. La crisis hídrica trae aparejado graves problemas ambientales económicos y sociales, que hoy atraviesa la realidad del sector. A su vez existe en el sector una larga disputa por el acceso a la tierra, al agua y en la garantía de derechos sociales como es el acceso a un hábitat digno. Por lo tanto, la población que habita en el territorio no irrigado del departamento de Lavalle ${ }^{1}$, al norte de la provincia de Mendoza, se considera un sector vulnerable de la sociedad mendocina, por las condiciones de pobreza, exclusión y la deficiencia habitacional, que en su devenir histórico ha desarrollado particulares formas de organizarse para resolver sus necesidades de habitar, despojados de recursos, de infraestructura y apropiados de un territorio tan hostil como es el área (semi) desértica.

\section{Herramientas Conceptuales}

La arquitectura es una disciplina que tiene gran protagonismo en el estudio del hábitat, ya que impacta de forma negativa o positiva- en la configuración del territorio y es un elemento que incide en la imagen visible de ese territorio. En este sentido frente al dinamismo que caracteriza al territorio, la arquitectura se despliega como intérprete de lo acontecido y refleja cómo se ha realizado la apropiación y organización del hábitat (Flores, 2004; Quintana Andrés, 2008).

Se comprende al territorio como una construcción social, como el resultado de la acción de un actor o un grupo humano sobre la naturaleza, donde se observa la dialéctica entre ambos. Raffestin lo define como "el espacio apropiado y valorizado -simbólica e instrumentalmente- por grupos humanos" (1980, p.129). Esta apropiación-valoración puede ser de carácter instrumental-funcional o simbólico-expresivo, donde en el primer caso se enfatiza la relación utilitaria y en el segundo se destaca como asiento simbólico-cultural de identidades individuales y colectivas (Giménez, 2001). Una de las características principales del territorio es su dinamismo, ya que no se comprende como una realidad acabada, sino que por el contrario, se encuentra en permanente construcción y transformación (Vallejos, 2008; Raffestin, 1980). En este sentido, Sánchez (1991) postula a la consideración del tiempo y el espacio para su análisis y comprensión, desde la articulación de aspectos sociales, económicos y ecológicos que repercuten en su configuración.

El quehacer de la arquitectura no implica de forma exclusiva la construcción de edificios-viviendas, donde prima una mirada reduccionista a lo técnico, a la materialización de un cobijo y -en algunos casos- a la resolución estética del habitar, sino que en un sentido más amplio, la arquitectura es una disciplina que permite relevar lo existente, visibilizar la materialización efectiva de otras formas no académicas de construir (como la producción social del hábitat según Ortiz, 2012; Romero, 2002) en la que se puede leer también la vida social, cotidiana y simbólica de la población. En este sentido, el

${ }^{1}$ El territorio involucra los distritos de Asunción, Lagunas del Rosario, San Miguel, San José y sólo la zona no irrigada de Jocolí y San Francisco. La población es de 3.536 personas que habitan de forma dispersa en el territorio. (Censo INDEC, 2010). 
autor Pallasmaa (2016) expresa que "la arquitectura tiene su origen ontológico en el acto de habitar" en el significado de la vida, específicamente en la vida cotidiana, y el autor remata con la afirmación de que "habitar forma parte de la propia esencia de nuestro ser y de nuestra identidad" (2016:16-18).

Dentro de la arquitectura y en relación con el hábitat rural, la vivienda proporciona información para entender los modos de vida y el funcionamiento cotidiano de las actividades productivas en ella desarrollados (Sánchez Quintanar y Jiménez Rojas, 2010). Además, mediante el estudio de la vivienda rural resulta posible indagar como se ha organizado el territorio en que se encuentra (Rapoport, 2003). La realización de "un tipo de habitación rural resulta, la mayoría de las veces, de una larga evolución; que resume la experiencia de generaciones rurales" (Demangeon, 1920 citado en Florido Trujillo 1996:29) ya que está influenciada por diferentes procesos territoriales, y, por lo tanto, "reúne un importante cúmulo de saberes atesorados de forma progresiva a lo largo del tiempo" (Florido Trujillo 1996:29).

Existen diversos autores que definen a esta línea de estudio como arquitectura vernácula o arquitectura rural, basadas principalmente en las tecnologías utilizadas y delimitadas en mayor medida a la relación rural-agrícola. Resulta preciso aclarar que lo vernáculo significa en latín esclavo nacido en la casa del dueño (Benavidez Solís, 1997:60) y hace referencia también a la arquitectura preindustrial ligado a lo indígena o nativo. En este sentido se considera que tanto lo vernáculo como lo rural tiende a confinar el abordaje de la realidad rural (y en especificidad a las zonas no irrigadas) a un conocimiento técnico-material de la arquitectura preindustrial y su valor patrimonial. En las últimas décadas hay estudios que refieren al hábitat rural desde y hacia sus habitantesusuarios, en el que se destacan también sus formas de organizarse, los constructores actuales, las mixturas de tecnologías y las connotaciones de la vida cotidiana como es la arquitectura doméstica (Kellett, 2002) o el espacio doméstico (Sanín Santamaría 2008), arquitectura sin arquitectos (Rudofsky, B., 1973; Turner, 1978) o la arquitectura popular (Conolly, 2011; Montaner JM, 2012).

La arquitectura popular es un campo de estudio y quehacer de la disciplina en la que (con o sin intervención de profesionales técnicos) la población resuelve el hábitat a partir de sus saberes o tradiciones tanto en la apropiación de la naturaleza como en la materialización de la vivienda. Según Romero Torres, J (2000:2) la arquitectura popular evidencia una forma de construir íntimamente ligada a creencias y prácticas colectivas de condición funcional, y asociadas a actividades concretas. El motivo principal por el cual se fomenta este tipo de arquitectura es la necesidad imperante de acceder a un hábitat digno, es decir que este modo de hacer arquitectura está movilizado por una lógica de producción habitacional sin fines de lucro que busca resolver una necesidad (que es habitar) y su satisfactor principal es la vivienda.

Resulta importante definir que "lo popular" son aquellas manifestaciones de los sectores desfavorecidos que habitan de las ciudades principalmente aquellos que se localizan en zonas periféricas, villas $\mathrm{u}$ asentamientos. También en esta categoría se encuentran los trabajadores rurales empobrecidos, campesinos, crianceros y pobladores indígenas. Según la Comisión por la memoria, los sectores populares no forman parte del discurso dominante, de los relatos históricos, y casi nunca son protagonistas de los procesos sociales y políticos. Sin 
embargo Baño (2004:37) escribe en este sentido que lo popular no es una categoría inerte, sino que incide, por distintos medios y con distinta fuerza, en la definición de la voluntad colectiva de una sociedad, que es el campo de la política. Para autores como Palma (1987) lo popular comprende también organización y cultura, es decir no solo es definido por la pobreza, sino que hacer referencia a la capacidad colectiva, las propuestas e iniciativas de la población (citado en Hernández García, J y Rojas Sánchez, E. 2014:190).

El campo de estudio de la arquitectura popular está estrechamente ligado a las diversas manifestaciones del hábitat popular. Algunos autores como Priscilla Connolly (2011:28) le llaman a este fenómeno el "paradigma latinoamericano del hábitat popular", quien afirma que es objeto de estudio desde 1970 en adelante, momento que cobra relevancia en los temas de discusión de organismos internacionales en Latinoamérica. En este sentido, desde la década del 70 el tema del hábitat popular cobra importancia en la agenda política, como afirma Miranda Gassull, V (2017:233): "a partir del impulso de organizaciones sociales de base en la temática, la conformación de redes latinoamericanas y al interior de la academia un revisionismo crítico de los devastadores resultados del modelo neoliberal, el rol profesional y la necesidad de repensar alternativas a la crisis habitacional de las ciudades latinoamericanas". Este trabajo pretende desentrañar a la arquitectura popular con cierto rigor científico, al menos en el diseño metodológico aplicado a zonas rurales no irrigadas.

Entonces desde la perspectiva de la arquitectura popular, se propone resignificar no solo los edificios-viviendas rurales, sino atender a los procesos sociales que se involucran en la producción habitacional, la estrecha relación con la naturaleza y la compleja trama territorial que se entreteje en las dinámicas rurales de climas áridos o (semi) desérticos.

Para el diseño metodológico desde la arquitectura popular, hay dos premisas de partida en este trabajo que son fundamentales expresar. Por un lado, en el hábitat rural de tierras secas no irrigadas se comprende a la vivienda como la relación irreductible entre la unidad doméstica y la unidad de producción (Pastor, 2005). Es decir, el espacio interior familiar se materializa hacia el espacio exterior donde se encuentran las actividades productivas, donde lo íntimo y lo externo están afincados en una sola interacción. Por otro lado, como la arquitectura que se investiga existe a priori a la intervención, se apela a una de las técnicas de mayor uso en el análisis de obras construidas como es el relevamiento arquitectónico. Se aplica para determinar el estado habitacional, calidad constructiva y valor estéticofuncional de la obra. Así mismo, en las zonas rurales resulta una técnica fundamental para dar cuenta de la materialidad existente, determinar la ubicación de las viviendas y también entrever los modos de vida asociados al carácter rural.

\section{Resultados: Procedimientos metodológicos aplicados al hábitat rural no irrigado.}

Las propuestas metodológicas que se presentan se centran en dos vías para abordar la configuración habitacional de asentamientos rurales no irrigados. Por un lado, el diseño metodológico basado en la relación naturaleza-cultura expresada por la articulación entre disponibilidad y uso de los bienes de la naturaleza, las actividades productivas que se realizan y las redes familiares. Por otro lado, se expone una metodología que se basa en una mirada integral del hábitat rural (determinada por cuatro dimensiones) atravesadas por las formas de organización 
social de la producción habitacional, que establece como resultado una particular relación entre el hábitatterritorio en pos de su organización social de base.

La primera metodología deriva del análisis de la interacción vivienda rural-territorio en el distrito de Lagunas del Rosario (ver Figura 2), donde se abordó a la vivienda rural como un hecho acoplado del territorio.

FIGURA 2: RELACIONES ENTRE LAS

CATEGORÍAS. SU ELECCIÓN RADICA EN SU

ÍNTIMA ARTICULACIÓN PARA LA

COMPRENSIÓN DE LA INTERACCIÓN VIVIENDA RURAL-TERRITORIO.



Fuente: Elaboración propia, 2016.

El territorio incide en la vivienda rural de acuerdo a la relación entre la disponibilidad y acceso a los bienes naturales (variable ecológica), a la composición familiar (variable social) y a las actividades productivas que se realizan (variable económica). La vivienda rural se adecúa y se adapta a las transformaciones del territorio, donde quedan de relieve aspectos materiales y simbólicos desde donde resulta factible observar los cambios y las estrategias que despliegan los pobladores para hacer frente a la vida diaria. También se consideraron diferentes escalas temporales y espaciales de análisis para comprender y enmarcar los hechos proyectados en el territorio que inciden en el hábitat de Lagunas del Rosario.

Los resultados obtenidos se tradujeron en la identificación de tres tipologías de asentamientos, que tienen que ver con la forma en que los pobladores se adaptan a las transformaciones del territorio:

1. Asentamientos familiares: compuestos por la presencia de entre 2 a 5 viviendas agrupadas donde la relación entre ocupantes es exclusivamente de lazos sanguíneos. Se localizan dispersos en el territorio y se dedican a la cría de ganado principalmente caprino. La presencia de la familia en el asentamiento resulta crucial para acceder al agua subterránea y contar con mano de obra para el desarrollo de majada. Son los asentamientos más antiguos registrados en el sector.

2. Asentamientos en Poblados, que se conforman por la presencia de más de 15 viviendas agrupadas. Por la mayor cantidad de gente viviendo en el sitio se dificulta la cría de ganado por la competencia por las pasturas y por ello sobresalen actividades vinculadas con artesanías, empleos estatales e incipiente turismo. Esta tipología se vio favorecida por la presencia de infraestructura y servicios estatales.

3. Asentamientos Solitarios, conformados por una única vivienda localizada de forma dispersa y aislada en el territorio. La mano de obra se limita a esa familia y sobresale la cría de ganado como actividad productiva principal. Estas viviendas se caracterizan por el desplazamiento de la vivienda conforme la mayor presencia de bienes naturales, principalmente agua y pasturas.

Luego, mediante el estudio de la vivienda rural como unidad de análisis también resultó factible leer la relación naturaleza-cultura. Desde el abordaje de la vivienda como 
objeto arquitectónico se comprendió el tipo y cualidades de los materiales constructivos empleados y su vinculación con los materiales disponibles en el medio y las estrategias bioclimáticas que se despliegan. Del estudio de las particularidades de la vivienda y los tipos de asentamientos surgieron nuevas dimensiones desde el trabajo de campo, que permitieron enriquecer los resultados obtenidos y que aportan datos que suman a la heterogeneidad de situaciones al interior del territorio. Estas dimensiones son: accesibilidad física, cantidad de gente en la vivienda o asentamiento, cercanía la infraestructura estatal, cantidad y tipo de actividades productivas, suelo (nivel de desertificación), vegetación (tipo) y formas de acceso al agua subterránea en el predio.

Mediante este estudio se pudo identificar los tipos de asentamiento en Lagunas del Rosario y las características diferenciales que presenta cada uno, donde de acuerdo a la relación entre las dimensiones se entiende el porqué de los asentamientos y las características que despliegan.

\section{La importancia de este diseño metodológico radica en}

la inclusión de las miradas y las acciones de los actores en la comprensión de la interacción vivienda ruralterritorio según la relación entre variables sociales, económicas y ecológicas. El uso de métodos cualitativos privilegió la construcción de datos mediante la participación de los diferentes actores que intervienen en el territorio recuperando las perspectivas, aspectos simbólicos y valoraciones que se ponen en juego frente a las transformaciones del territorio y las estrategias que despliegan los pobladores para la construcción del hábitat. Estas perspectivas puestas en tensión con datos provenientes desde diversas fuentes documentales permitieron, desde el método de análisis de contenido, fortalecer, enriquecer y matizar las acciones y estrategias vinculadas con el acceso y disponibilidad de bienes naturales, las redes familiares y las actividades productivas que se realizan dentro de la construcción del hábitat.

La segunda propuesta se basa en un procedimiento metodológico (Cano, A. 2012:28) referido a los modos concretos en que se utilizan las técnicas elegidas en función del contexto real en que se harán: es decir, cómo se aplican las técnicas seleccionadas en un determinado grupo y/o en un tiempo específico. Por lo general se utiliza una triangulación de técnicas para obtener los datos necesarios, pero resulta importante también establecer un marco de estudio que procure una investigación activa y democratizadora comúnmente denominada IAP-investigación acción participación (Campos, 1990). La realidad habitacional que existe en las poblaciones rurales semidesértico y/o no irrigada por lo general es crítica, lo que implica una intervención y/o transformación activa e inminente. Este es el caso de estudio propuesto, en el que la población tiene un déficit habitacional en un $93 \%$ sobre el total de la población (INDEC, 2010), marcando un grave estado habitacional. Entonces, desde este plano la investigación-acción deja de ser un proceso neutral de comprensión y práctica, y se convierte en un proceso crítico de intervención y reflexión. Es un proceso de indagación y conocimiento, un proceso práctico de acción y cambio, y un compromiso ético de servicio a la comunidad (Montero, 2006).

La arquitectura popular asume en la IAP un método dialógico que permite, por un lado, definir y detectar las condiciones habitacionales, pero también posibilita revelar el entramado social que se establece entorno al hábitat en un marco de espacio-tiempo construido conjuntamente con el rol activo de sus pobladores/as para pensar posibles soluciones. Esta metodología se aplicó en el distrito de La Asunción, en Lavalle, caso de estudio que 
permite relevar algunas variables e indicadores que son necesarios identificar tanto las condiciones habitacionales, como poner en valor procesos sociales que son llevados a cabo por sus pobladores, como también las posibles transformaciones que son prioritarias para sus pobladores.

Como se expuesto, la segunda metodología propuesta deriva del análisis de la interacción organizaciónhábitat-territorio en el distrito de Asunción, donde se aborda al hábitat como un componente esencial "en" y “del” territorio. En este sentido, el territorio y el hábitat están vinculados por los diferentes modos de organizarse (variable social) en torno al sistema de producción habitacional (variable socioeconómica) que cada grupoactor desarrolla y los componentes que resultan de esta interacción (variable física-ecológica). Ver Figura N³. El marco espacio-temporal es fundamental para comprender los procesos de producción habitacional de estudio, no sólo como fruto del esfuerzo y la gestión de los propios actores involucrados, sino para determinar las fuerzas sociales que se pugnan respecto al acceso de un hábitat digno y la heterogeneidad territorial de las zonas rurales no irrigadas. Es decir, determinar cómo se entrelaza a la problemática habitacional que afecta a la población desde procesos y no sólo hechos, pero a su vez para determinar cuáles son las condiciones sociales que llevaron al estado del hábitat de zonas vulnerables.

\section{FIGURA 3: METODOLOGÍA APLICADA AL} HÁBITAT RURAL.

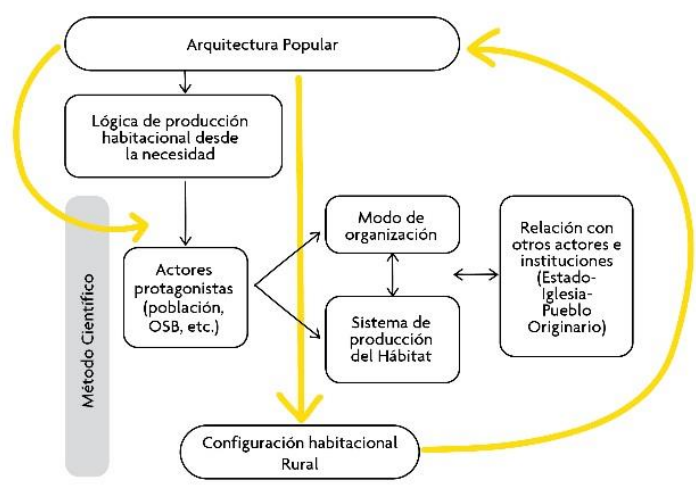

Fuente: Elaboración propia, 2017.

Las variables principales de esta propuesta metodológica son el modo de organización y relación con el territorio (a) y el sistema integral en la producción del hábitat (b).

\section{a. Modo de organización y la relación con el territorio}

Se determina por los actores que se reconocen en el proceso y la relación que tienen con el rol del Estado estableciendo diferentes modos de producir el hábitat. Algunos autores como Rodríguez y Di Virgilio (2007:18) diferencian la autoproducción, autogestión, autoconstrucción y producción por organismos sin fines de lucro, donde las universidades han tenido un rol destacado en esta última. Se exponen tres modalidades que se pueden dar de forma simultánea, en diferentes tiempos o solo desarrollarse una sola fase de estos procesos.

1. Autoconstrucción: obra realizada por mano de obra no remunerada de forma individual (autoayuda) y/o familiar - colectiva 
2. Autoproducción: el control de la producción habitacional se hace en todo el proceso, no sólo en la construcción, sino en el diseño, ejecución, administración, evaluación, y mantenimiento. Puede ser realizada por familias o individuos; o de forma comunitaria, colectiva y organizada. También pueden asociarse a toras entidades como organismos sin fines de lucro, universidades, etc.

3. Autogestión: Por lo general esta fase está ligada a las anteriores, en donde funda el poder autogestivos en la disputa por una redistribución material, el reconocimiento territorial y/o la diversidad cultural.

\section{b. El Sistema de Producción del Hábitat.}

Se entiende a la construcción de la vivienda como un sistema de producción dentro de los procesos sociales del hábitat que involucran los siguientes componentes: tierra o suelo, servicios sociales y equipamientos básicos, infraestructura, materiales y componentes, asistencia técnica, capacidad de pago, financiamiento, movilización de recursos y el fortalecimiento de la economía popular, la fuerza de trabajo y la participación de los usuarios.

La vivienda es la menor unidad de análisis se entiende como la principal condición material en los procesos de autoproducción del hábitat, atravesada por un lado, por el valor de uso de la vivienda (en cuanto a satisfacción de necesidades humanas), y el valor de cambio dado por la fuerza de trabajo implicada en la misma y su valor monetario que adquiere en el mercado. (Rodríguez et al, 2007:21)

\footnotetext{
A modo tal de realizar un análisis sistémico del hábitat se determinan las siguientes variables e indicadores para su orden escalar y material:
}

1. Unidad doméstica establecida por la vivienda como elemento material principal. Esta es la menor escala de análisis. Algunos indicadores son: tamaño-ubicación de la vivienda-puesto, dimensiones de la vivienda, locales usados, materiales y, distancia a servicios y accesos. También se incluyen los modos de construcción y la tecnología utilizada como conocimientos y saberes propios de la población.

2. La relación de la vivienda con las actividades económicas. (Se proponen dos indiciadores: La vivienda productiva relacionada a las unidades donde predomina alguna actividad ganadera y/o agrícola; y las viviendas pluriactivas donde se diversifica la matriz de actividades económicas de sustento)

3. La relación de la vivienda con la infraestructura y equipamiento y las formas de acceso al suelo (tenenciadominio, equipamiento de salud, educación, uniones vecinales, disponibilidad de agua-redes...etc).

4. La relación entre el hábitat y el territorio: como resultado de las dimensiones anteriores, se produce una determinado forma de establecerse la dialéctica entre la configuración del hábitat y la apropiación del territorio. Como se puede observar en la Figura $\mathrm{N}^{\circ} 4$, las diferentes escalas de trabajo que constituyen el sistema del hábitat son atravesados por los modos de organización expuestos anteriormente (se puede cumplir un solo modo o los tres) y la relación con los actores principales que promueven (o no) la producción habitacional. 
FIGURA 4: ESQUEMA DE VARIABLES E INDICADORES HÁBITAT RURAL.

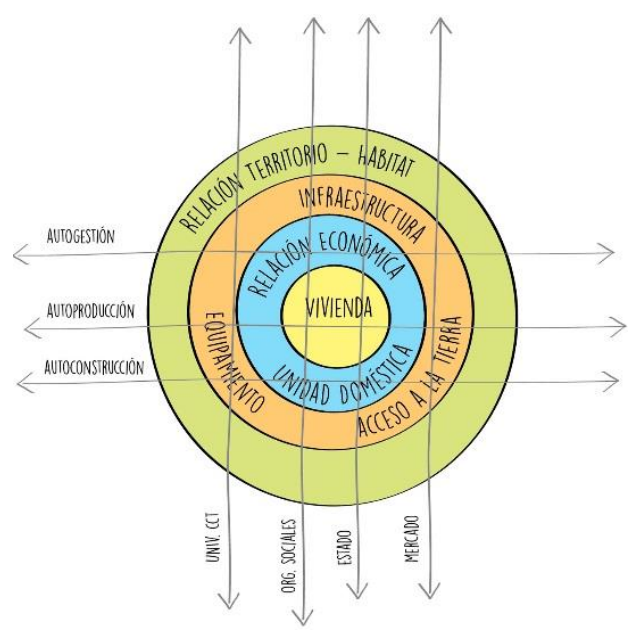

Fuente: Elaboración propia 2017.

Como resultado del uso de esta metodología se identifican/establecen patrones o tipologías de asentamiento que se definen como:

1. El caserío agrupado de más de 20 viviendas: determinados por la actividad económica (plural), cantidad de puestos concentrados (distancia menor a $1 \mathrm{~km}$ entre sí), disponibilidad u acceso a equipamiento de salud y educación (distancia menor a $2 \mathrm{~km}$ ) y la cercanía a la conectividad vial para establecer un patrón nucleado poblacional. En el caso de Asunción se puede observar autoproducción familiar en viviendas y autoproducción colectiva en equipamientos e infraestructura. Los procesos de autoproducción habitacional han conllevado a la autogestión del territorio en vínculo con otras comunidades y distritos del área.

2. El patrón de asentamiento mixto o de transición se establece por actividad económica (plural y ganadera), cantidad de puestos concentrados (menos de 20 a una distancia mayor a $1 \mathrm{~km}$ entre sí), disponibilidad $\mathrm{u}$ acceso a equipamiento de salud y educación (distancia mayor a
$2 \mathrm{~km})$ y la cercanía a la conectividad vial. Se puede observar el autoconstrucción como modo predominante de realizar las viviendas, y los equipamientos comunitarios en fase o de diseño o de ejecución desde la autoproducción colectiva.

3. Mientras que las viviendas dispersas, se obtienen como resultado de la distancia entre viviendas (mayor a 5 $\mathrm{km}$ ) en el territorio, que no cuentan con equipamiento y/o accesos viales cercanos. Sólo se observa la autoconstrucción y autoproducción individual y/o familiar. La base familiar es el núcleo organizacional de las familias crianceras de zonas no irrigadas

La importancia de esta metodología radicó en la consideración de la dimensión social como clave para comprender la construcción del hábitat y las formas de organización social desde el abordaje del concepto de autoproducción. No obstante, el protagonismo de la dimensión social, se cruzaron datos con las dimensiones sociales y ecológicas para abordar de forma más precisa al territorio, es decir considerando al binomio naturalezacultura.

La dispersión de los asentamientos rurales y la vivienda no resultan como islas desiertas disgregadas en el espacio, sino todo lo contrario, en esa dispersión, en las unidades productivas y domésticas radica el control territorial, la base de poder y la organización primaria de estas poblaciones.

\section{Intersecciones de las propuestas metodológicas en el abordaje del hábitat de las tierras secas.}

En ambas propuestas metodológicas el relevamiento arquitectónico fue una clave diferencial para el abordaje 
y construcción de datos respecto a las viviendas y los asentamientos. Este relevamiento implicó no sólo el abordaje del edificio, sino que también datos vinculados con aspectos sociales, económicos y ecológicos. Es decir que se propició una mirada que supera la visión objetual del edificio construido (que domina la disciplina arquitectónica) para incorporar datos desde el territorio, tanto materiales como simbólicos. En este sentido, se coincide con Rapoport (1968) cuando expresa que "no se puede ver la vivienda aislada de su asentamiento, sino que ha de ser considerada como una parte de todo el sistema social y espacial que relaciona la casa, el modo de vida, el asentamiento y hasta el paisaje......la geografía y la arquitectura han separado, habitualmente, el estudio de la vivienda y el asentamiento, sin embargo, la necesidad de examinarla como parte de un sistema más amplio confirma que la vivienda tiene poco sentido fuera de su entorno y de su contexto" (Rapoport, 1968: 93).

Otra de las grandes coincidencias entre ambas propuestas tiene que ver con el abordaje de la vivienda como unidad de habitación y de producción. $\mathrm{La}$ consideración de esta dualidad permite comprender al objeto arquitectónico y las actividades que alberga en el interior y exterior como un todo, donde cada elemento tiene su sentido y su rol dentro del hábitat rural. Esto resulta interesante ya que en las políticas habitaciones es común el abordaje de la vivienda como objeto construido, donde quedan relegados a un segundo plano su vinculación con aspectos ecológicos y económicos, originados principalmente por la falta de datos respecto a las zonas rurales y porque muchas veces se piensa lo rural desde una mirada exclusivamente urbana (Dalla Torre et al, 2017). Esto se origina ya que se considera al objetovivienda como respuesta a una necesidad básica. En este sentido, se coincide con Echeverría (2011) cuando enuncia que "la vivienda no es una necesidad humana, sino un satisfactor, una respuesta, oportunidad y desenlace diversificados de lo individual, familiar y grupal; que se despliega desde una variedad de resoluciones, significaciones, funciones, usos y actividades; según diferentes etnias, grupos, actores, realidades, circunstancias, necesidades, valoraciones, imaginarios, costumbres, prácticas; gestadas mediante procesos múltiples" (2011:4).

El uso de metodologías cualitativas para la construcción de datos resultó en un aspecto fundamental en ambas propuestas, donde se incluye a la población local como actores claves en la construcción del hábitat y el territorio. A su vez, la metodología empleada confiere carácter científico tanto a la intervención en comunidades aisladas-vulnerables, como así también a los procesos de trasformación de los diferentes componentes del hábitat. El dialogo entre saber y conocimiento son posibles gracias a la labor científica pero principalmente al cuidado metodológico empleado, el respeto a la diversidad y el reconocimiento de los actores como protagonistas de su trasformación (no sólo en la fase consultiva).

Finalmente, resulta una herramienta clave la consideración de diferentes escalas espaciales y temporales en el análisis de los procesos de territorialización, para comprender las transformaciones y adecuaciones emergentes de la interacción territoriovivienda rural y poder contextualizar las acciones y valores que despliegan los pobladores en la construcción del hábitat. Soja plantea el abordaje multiescalar desde la meso escala como una mirada "desde arriba" hacia lo local, donde se logran ver las redes y flujos existentes en el territorio, para posteriormente trasladarse a la micro escala, para poder apreciar así, las relaciones in situ de lo 
local, qué es fundamental para analizar y entender la producción social que se genera (2004 citado en González 2011:10).

\section{Conclusiones}

Al estudiar el hábitat en zonas rurales no irrigadas, la primera dificultad que se encuentra es la escasa o nula información que hay para definir un diseño metodológico afín, (principalmente) desde la disciplina arquitectónica. Por lo general, el criterio para definir lo "rural" es considerado como todas aquellas localidades con menos de 2000 habitantes, sin embargo, este dato no revela que la ruralidad es diversa, heterogénea y existe una dificultad logística para relevar datos dado por las distancias de kilómetros que hay entre unos y otros pobladores. Entonces, lo rural y principalmente lo rural no irrigado, implica para su estudio, una elaboración meticulosa de los procedimientos metodológicos a seguir. En este sentido, a través del desarrollo de las investigaciones doctorales, la puesta en práctica de la arquitectura como disciplina fundante para la investigación y construcción de información de poblaciones rurales (semi) desérticas, resultó en una decisión efectiva que posibilita analizar y destacar la complejidad rural.

Hay que subrayar que un abordaje interdisciplinar amplía e integra la mirada sobre el objeto de estudio, sin embargo, este artículo quiere destacar el rol de la arquitectura en los procesos metodológicos (inter) disciplinarios y la utilidad de las técnicas que se emplean para el trabajo de campo de ésta. La arquitectura combina una relación entre aspectos materiales y simbólicos, que permiten reflexionar y comprender la configuración del hábitat de un determinado grupo social. La vinculación entre la materialización de la obra como un aspecto físico y cuantificable, el uso tecnológico pensado según la disponibilidad de bienes naturales, la estructura socioeconómica y la organización social en torno al sistema de producción del hábitat, son capaces de vincularse desde la arquitectura y proponer resultados en torno a la diversidad de formatos que se presentan en poblaciones rurales no irrigadas de baja densidad. Por lo tanto, la relación entre los diversos componentes que se estudian en la arquitectura permiten aproximarse a un nivel escalar local-especifico con gran nivel de detalle, comprender los hechos materiales y organizativos desde la realidad actual, pero anclada en una trayectoria espacio-temporal.

A modo de respuesta del interrogante de este artículo, el aporte metodológico presentado, constituido con herramientas y técnicas usualmente aprovechadas en la arquitectura permite abordar el estudio del hábitat rural de tierras secas y valorizar el entramado territorial de estas poblaciones.

Resultan interesantes las coincidencias de las propuestas metodológicas expuestas que tienen que ver con la comprensión de los diferentes factores que inciden en la construcción del hábitat. La articulación de estas dimensiones considerando una mirada (inter)disciplinar facilita una respuesta que se aproxima a la realidad de la población local y por tanto la posibilidad de que las políticas públicas y acciones hacia el territorio y la arquitectura resulten más coherentes con el ámbito cultural y ecológico de inserción.

\section{Bibliografa}

Baño, R. (2004). Los sectores populares y la política: una reflexión sociohistórica. Política, (43), 35-55. Disponible en: <http://revele.com.veywww.redalyc.org/articulo.oa?id=64504303> ISSN 0716-107

Benavidez Solís, J. (1997). La Arquitectura vernácula: una memoria rota. Revista PH, (20), 60-64 
Campos, Armando, (1990). Investigación Participativa: Reflexiones acerca de sus fundamentos metodológicos y de sus aportes al desarrollo social. Ponencia presentada en seminario Internazionale e la Ricera Sociale, Roma. Italia.

Cano, A. (2012). La metodología de taller en los procesos de educación popular. Revista Latinoamericana de Metodología de las Ciencias Sociales, 2 (2), 22-51. En Memoria Académica. Disponible en: http://www.memoria.fahce.unlp.edu.ar/art_revistas/pr.5653/pr.5653.pdf

Censo Nacional de Población, Hogares y Viviendas (2010). Argentina. Disponible en https://www.indec.gov.ar/nivel4_default.asp?id_tema_1=2\&id_tema_2=4 1\&id_tema_3=135

Chiavazza, Horacio (2008). Cambios ambientales y sistemas de asentamiento en el árido normendocino. Arqueología en los paleocauces del Río Mendoza (Tesis doctoral). Universidad Nacional de La Plata. Buenos Aires, Argentina.

Connolly, Priscilla. (2011). La ciudad y el hábitat popular: Paradigma latinoamericano. Biblioteca Catedra Sur de la Universidad Autónoma Metropolitana y Universidad General de Sarmiento. 41 paginas-

Dalla Torre, Julieta; Sales, Romina; Esteves, Matías; GhilardiI, Matías (2017). "Las particularidades de las fronteras urbano-rural en el ordenamiento territorial. VI Seminario Internacional de Ordenamiento Territorial. Mendoza, UNCUyo.

Echevarría, Clara (2011). "Hábitat del habitar, como territorio étnico, grupal y socialmente significado". Construcción Social Del Hábitat. Recuperado

https://construccionsocialdelhabitat.files.wordpress.com/2011/04/hc3albi tat-del-habitar.pdf

Flores, Fernando (2004). "La arquitectura como territorio". Cuaderno Arteoficio $\mathrm{N}^{\circ} 3$, pp. 7-12.

Florido Trujillo, Gema (1996). Hábitat rural y gran explotación en la Depresión del Guadalquivir. Sevilla: Consejería de Obras Públicas y Transporte, Junta de Andalucía.

Giménez, Gilberto (2001). "Cultura, territorio y migraciones. Aproximaciones teóricas”. Alteridades, Vol. 11, №22, pp. 5-14

González, Alejandro (noviembre, 2011). "Nuevas percepciones del territorio, espacio social y tiempo. Un estudio desde los conceptos tradicionales (clásicos) hasta su concepción en el siglo XX". VI Jornadas de Jóvenes Investigadores. Instituto de Investigaciones Gino Germani, Facultad de Ciencias Sociales, Universidad de Buenos Aires.

Gudiño, E. (2017). Ordenar el territorio: Un desafío para Mendoza. Mendoza: EDIUNC, Mendoza.Argentina.

Ivars, J. D. \& Larsimont, R. (2015). Saberes tradicionales y nuevas tecnologías agrarias e industriales: violencia epistémica en el acceso y el manejo del agua en Mendoza-Argentina. Alternativa. Revista de Estudios Rurales, 1(3), 1-29

Kellett, Peter. (2002). The construction of home in the informal city. [En línea]. Journal of Romance Studies. 2(3): 17-31, 2002. ISSN 1752-2331. Disponible en: http://dx.doi.org/10.3167/147335302782484720.

Ley 8051/2009. Ley de Ordenamiento Territorial de Mendoza.

Martín, F. (2009) "Las transformaciones recientes en la agricultura de oasis en Mendoza, Argentina. Una aproximación al caso de la reestructuración vitivinícola desde la economía política de la agricultura". Tesis de Maestría en Estudios Sociales Agrarios. FLACSO- Argentina. Recuperado de Flacso Andes:

http://flacsoandes.org/dspace/bitstream/10469/1805/1/Tesis_Facundo_Ma rtin.pdf
Montaner, Josep María. (2012). Arquitectura y Crítica en Latinoamérica. Buenos Aires Nobuko.

Montaña, E; L. Torres, E. M. Abraham, E. Torres y G. Pastor. (2003). En colaboración: S. Urbina y M. E. Fusari. Los espacios invisibles. Subordinación, marginalidad y exclusión de los territorios no irrigados en las tierras secas de Mendoza, Argentina. Enviado a la revista Región y Sociedad de El Colegio de Sonora, Hermosillo, Sonora, México, 2003.

Montaña, Elma. (2008). Las disputas territoriales de una sociedad hídrica. Conflictos en torno al agua en Mendoza, Argentina. Revista Iberoamericana de Economía Ecológica Vol. 9: 1-17. Disponible en URL: http://www.redibec.org/IVO/rev9_01.pdf

Montero, Maritza. (2006). Hacer para transformar: el método en la psicología comunitaria -1a ed. - Buenos Aires: Paidós, 2006. 372 p. (Tramas sociales) ISBN 950-12-45357

Miranda Gassull, Virginia (2017). El hábitat popular. Algunos aportes teóricos de la realidad habitacional de sectores desposeídos. Territorios, [S.1.], n. 36, ene. 2017. ISSN 2215-7484. Disponible en: doi:http://dx.doi.org/10.12804/revistas.urosario.edu.co/territorios/a.4440.

Ortiz Flores, E. (2012). Producción Social de la vivienda y el hábitat. Bases conceptuales y correlación con los procesos habitacionales. Edición Hábitat International Coalition. HIC.

Pallasmaa, Juhani. (2016). Habitar. Barcelona, España, Edición Gustavo Gili, SL. (10-44)

Palma, D. (1987). "La informalidad, lo popular y el cambio social". Lima: Desco, citado en Hernandez, Jaime y Rojas, Edisa. (2004) "Lo singular como alternativa conceptual y metodología para la construcción socioespacial de la ciudad" Revista Cuadernos de Vivienda y Urbanismo, Vol. 7 No. 14 julio-diciembre

Pastor, Gabriela (2005). Patrimonio, vivienda y agua en el paisaje del noreste mendocino. En: Fernández, A. \& E. M. Abraham (Eds.) El agua en Iberoamérica. Uso y gestión del agua en tierras secas. Pp. 79-92.

Raffestin, C. (1980). Por una geografía del poder. Traducción y notas Yanga Villagómez Velázquez. México: El Colegio de Michoacán.

Rapoport, Amos (1962). Vivienda y cultura. Barcelona: Gustavo Gili.

Rapoport, Amos (2003). Cultura, arquitectura y diseño. Barcelona: Edicions UPC.

Rodríguez, Adrián y Meneses, Javier (2011) “Transformaciones rurales en América Latina y sus relaciones con la población local”. Santiago: $C E P A L-$ $O N U$.

Rodríguez, M. Carla C., Di Virgilio M., Procupez V., Vio M., Ostuni F., Mendoza M., \& Morales B. (2007). Producción social del hábitat y políticas en el Área Metropolitana de Buenos Aires: historia con desencuentros. [Documento de trabajo del Instituto de Investigaciones Gino Germani, Facultad de Ciencias Sociales. UBA].

Roig, Fidel; Roig, Arturo; Roig, Mario; Roig, Virgilio \& Roig, Enrique (1999). Guanacache. Fidel Roig Matóns, pintor del desierto. Mendoza: EDIUNC.

Romero, Gustavo (2002). La producción social del hábitat. Reflexiones sobre su historia, concepciones y propuestas. En Ortiz Flores, E. y Zarate, L. (Comps.), Vivitos y coleando. 40 años trabajando por el hábitat popular de América Latina (pp. 70-79). México: UAM.

Romero Torres, Justo. (2000) Memoria y arquitectura popular. En: Gazeta de Antropología, № 16, 2000, Artículo 26.

Rosales, Diego (1877). "Historia General del Reyno de Chile, Flandes Indiano". En: Revista de la Junta de Estudios Históricos de Mendoza III. Mendoza. 
Rudofsky, Bernard. (1973). Arquitectura sin arquitectos. Buenos Aires: Editorial Universitaria.

Sánchez, E. (1991). Espacio, economía y sociedad. España: Siglo XXI Editores S.A., 1991

Sánchez Quintanar, C. \& Jimenez Rojas, E. (2010). "La vivienda rural. Su complejidad y estudio desde diversas disciplinas". Revista Luna Azul, N³0, pp. 174-196

Sanín Santamaría, Juan Diego. (2008). Hogar en Tránsito: Apropiaciones domésticas de la vivienda de Interés Social (VIS) y Reconfiguraciones del sentido de hogar. Antipoda. Revista de Antropología y Arqueología, (7), 31-61. Retrieved December 01, 2017, from http://www.scielo.org.co/scielo.php?script=sci_arttext\&pid=S1900$54072008000200004 \& \operatorname{lng}=$ en\&tlng=es.

Secretaría de Ambiente y Ordenamiento Territorial (2017). Plan Provincial de Ordenamiento Territorial (PPOT). Gobierno de Mendoza.

Teubal, M. 2001. «Globalización y Nueva Ruralidad en América Latina» en Giarracca, Norma. ¿Una nueva ruralidad en América Latina? Argentina, CLACSO. Disponible en http://bvsde.org.ni/clacso/publicaciones/UnanuevaruralidadenAmericaLati na.pdf\#page $=39$

Turner, J. F.C. (1978). "Housing in three dimentions: Terms of reference for the housing question redefined" / Publicado en: "Bromley R. (Ed.) "The urban informal sector Critical perspectives on employment and housing policies" / World Development, Vol. 6, No. 9/10 / Pergamon Press / Oxford.

Vallejos, Clara (2008). "Levantar la voz. Tensiones en los conflictos por la tierra en Santiago del Estero". IX Congreso Argentino de Antropología Social. Facultad de Humanidades y Ciencias Sociales, Universidad Nacional de Misiones. Misiones, Argentina

Vicuña Mackena, Benjamín (1856). Páginas de mi diario durante tres años de viajes. Santiago de Chile: Imprenta del Ferrocarril.

Quintana Andrés, Pedro (2008). "El hábitat y la vivienda rural en Canarias: Las transformaciones históricas de un espacio social". Rincones del Atlántico, $\mathrm{N}^{\circ} 5$, pp. 10-79. 
